\title{
Improving mental health care transitions for children and youth: a protocol to implement and evaluate an emergency department clinical pathway
}

Mona Jabbour 1,2,3* S. Reid 1,2,3, C. Polihronis 3,4, P. Cloutier ${ }^{3,5}$, W. Gardner ${ }^{5,6}$, A. Kennedy ${ }^{3,7}$, C. Gray 3,8,9 , R. Zemek ${ }^{1,2,10}$, K. Pajer ${ }^{8,9}$, N. Barrowman ${ }^{10,11}$ and M. Cappelli $i^{3,5,8,12}$

\begin{abstract}
Background: While the emergency department (ED) is often a first point of entry for children and youth with mental health $(\mathrm{MH})$ concerns, there is a limited capacity to respond to $\mathrm{MH}$ needs in this setting. Child $\mathrm{MH}$ systems are typically fragmented among multiple ministries, organizations, and providers. Communication among these groups is often poor, resulting in gaps, particularly in transitions of care, for this vulnerable population. The evidence-based Emergency Department Mental Health Clinical Pathway (EDMHCP) was created with two main goals: (1) to guide risk assessment and disposition decision-making for children and youth presenting to the ED with MH concerns and (2) to provide a streamlined transition to follow-up services with community MH agencies (CMHAs) and other providers. The purpose of this paper is to describe our study protocol to implement and evaluate the EDMHCP.
\end{abstract}

Methods/design: This mixed methods health services research project will involve implementation and evaluation of the EDMHCP in four exemplar ED-CMHA dyads. The Theoretical Domains Framework will be used to develop a tailored intervention strategy to implement the EDMHCP. A multiple baseline study design and interrupted time-series analysis will be used to determine if the EDMHCP has improved health care utilization, medical management of the $\mathrm{MH}$ problems, and health sector coordination. The primary process outcome will be the proportion of patients with $\mathrm{MH}$-specific recommendations documented in the health record. The primary service outcome will be the proportion of patients receiving the EDMHCP-recommended follow-up at 24-h or at 7 days. Data sources will include qualitative interviews, health record audits, administrative databases, and patient surveys. A concurrent process evaluation will be conducted to assess the degree of variability and fidelity in implementation across the sites.

Discussion: This paper presents a novel model for measuring the effects of the EDMHCP. Our development process will identify how the EDMHCP is best implemented among partner organizations to deliver evidence-based risk management of children and youth presenting with $\mathrm{MH}$ concerns. More broadly, it will contribute to the body of evidence supporting clinical pathway implementation within novel partnerships.

Trial registration: ClinicalTrials.gov (NCT02590302)

Keywords: Clinical pathway, Mental health, Emergency department, Pediatric, Risk assessment, Service integration, Care transitions, Implementation, Scoring tools

\footnotetext{
* Correspondence: jabbour@cheo.on.ca

'Division of Emergency Medicine, Department of Pediatrics, Children's

Hospital of Eastern Ontario, Ottawa, ON K1H 8L1, Canada

${ }^{2}$ Faculty of Medicine, Department of Pediatrics, University of Ottawa, Ottawa,

Canada

Full list of author information is available at the end of the article
} 


\section{Background}

Approximately $80 \%$ of children and youth with mental health $(\mathrm{MH})$ problems do not receive any $\mathrm{MH}$ services [1-3]. However, this seems to be impacting emergency departments (EDs), which are a commonly used access point for care. Due to the scarcity of child and youth $\mathrm{MH}$ services, EDs have increasingly become the place that families present for help. In its baseline scorecard for child and youth $\mathrm{MH}$ within Ontario, Canada, the Institute for Clinical Evaluative Sciences (ICES) reported marked annual increases in ED presentations between 2009 and 2012 among youth for anxiety, mood, and substance abuse disorders [4]. At one Canadian ED, visits for $\mathrm{MH}$ concerns among children and youth increased, more than $50 \%$ increase from 2010 to 2011 [5], far exceeding the $15 \%$ increase in overall patient visits documented in the hospital administrative database.

Although families attempt to access treatment and services in these settings, EDs are ill equipped to manage these pediatric $\mathrm{MH}$ problems. EDs typically lack standardized $\mathrm{MH}$ screening tools and pediatric $\mathrm{MH}$ expertise. In addition, with the complex and fragmented $\mathrm{MH}$ systems across North America [3, 6], many EDs lack defined, reliable, and integrated referral processes to appropriate community resources. Therefore, ED clinicians have difficulty identifying the appropriate $\mathrm{MH}$ services to which to refer patients for follow-up care [7].

To address these concerns, a group of Canadian hospital and community-based professionals was commissioned by the Provincial Council on Maternal Child Health in Ontario to create a clinical pathway, known as the Emergency Department Mental Health Clinical Pathway (EDMHCP) for children and youth presenting with $\mathrm{MH}$ problems (see Fig. 1). The EDMHCP is designed to (i) improve early identification and intervention for CYMH issues, (ii) provide more timely access to community $\mathrm{MH}$ services, and (iii) reduce service gaps for vulnerable children and youth. It defines a standardized approach to risk assessment and disposition decision-making and while improving access to appropriate needs-based $\mathrm{MH}$ care [8]. Finally, the EDMHCP aims to provide a seamless transition of care for children/youth and caregivers between the ED, outpatient hospital services and community $\mathrm{MH}$ agencies (CMHA), with tailored linkages to community resources through the HEADS-ED screening tool [9] incorporated within the pathway.

The overall aim of our project is to implement and evaluate impact of the EDMHCP in specific settings and to evaluate the implementation process. The purpose of this paper is to document the protocol for this 3-year mixed methods health services research project. Research objectives for this study are to:
1) Implement the EDMHCP using a theory-driven, evidence-based approach. The pathway will be implemented in four dyads of organizations, each dyad comprising an ED and an associated CMHA.

2) Evaluate clinical pathway effectiveness through measurement of process and service outcomes. Our hypothesis is that a successfully implemented EDMHCP will lead to improved process and service outcomes.

3) Conduct a process evaluation of the pathway implementation strategy to assess the fidelity of the intervention delivered against the outcomes observed.

\section{Methods/design}

The protocol for this $\mathrm{MH}$ project was adapted from a recent ED clinical pathway implementation protocol [10]. Collaborating with site partners, a theory-based and tailored intervention strategy will be developed to implement the EDMHCP within each ED-CMHA dyad. A concurrent process evaluation will be conducted to assess the implementation strategy. For this protocol, we completed the CONSORT 2010 checklist for randomized trials [11].

\section{Sampling \\ Setting and site selection}

To assess EDMHCP feasibility in various settings, implementation will occur in a staggered process in four Ontario exemplar ED-CMHA dyads with different patient populations and workflows that do not currently utilize clinical pathways. Our exemplar ED sites include a pediatric specialized center with an annual patient census of 70,000, a high-volume community urban hospital (annual patient census 75,000), a low-volume rural hospital (annual patient census 25,000), and a high-volume general hospital in a smaller community (annual patient census 60,000).

\section{Inclusion/exclusion criteria}

To begin, commitment to the implementation intervention by an administrative lead on behalf of each hospital and CMHA will be required. The EDMHCP has specific inclusion/exclusion criteria for patients presenting to the ED, which may be modified at each site based on organizational requirements. Inclusion criteria for patient participants include (1) ages $\geq 6$ and $<18$ years, (2) ED presentation within a selected 8-month implementation period, (3) MH presenting complaint, and 4) proficiency in English or French. Exclusion criteria include (1) highest priority triage designation (resuscitation), (2) medically unstable patients, (3) intubation/intensive care required, and (4) direct admission to hospital for ongoing medical management and observation. Patients who are stable but require medical management 


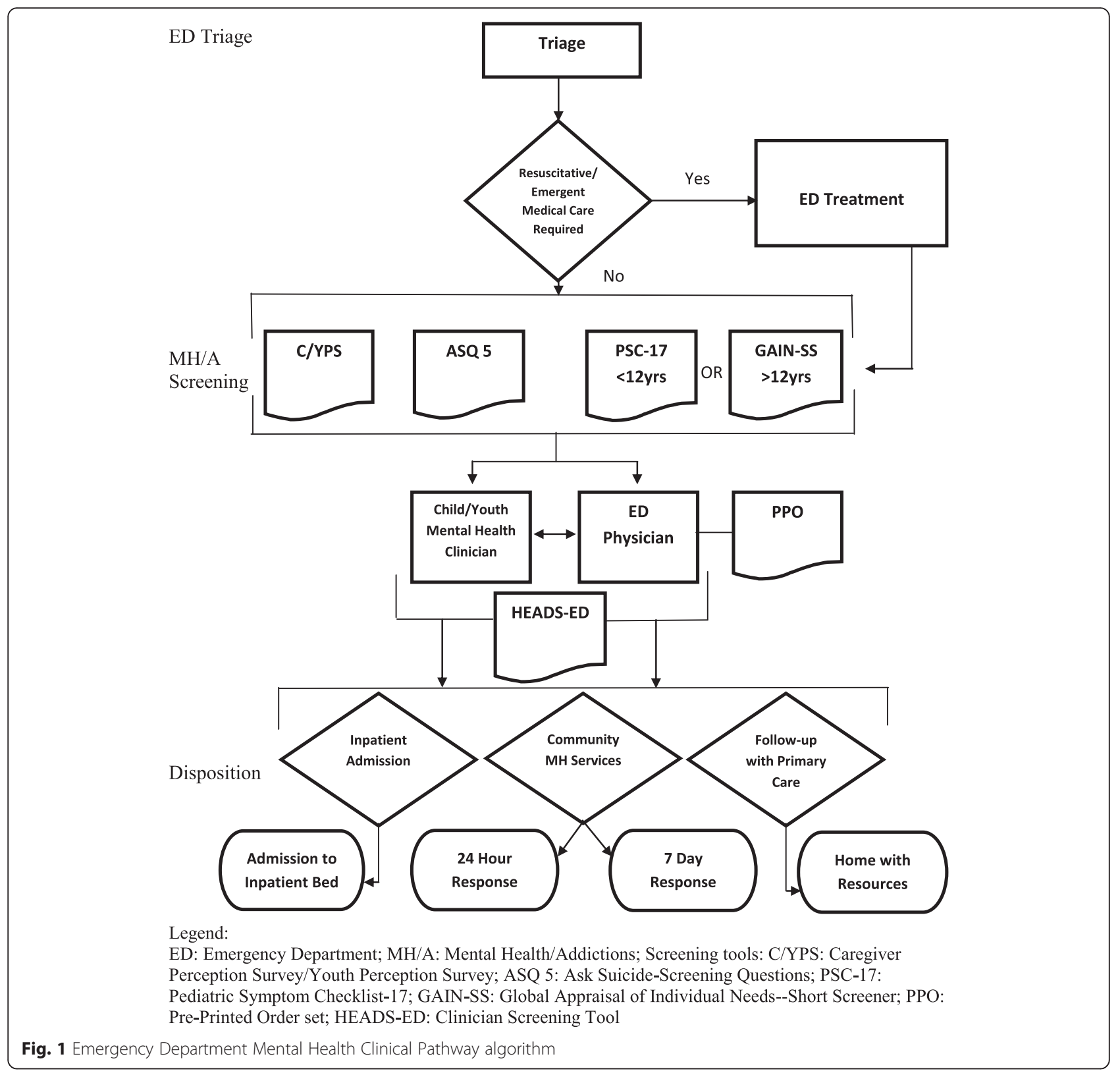

are eligible for the pathway once they are clinically able to participate in a $\mathrm{MH}$ assessment in the ED (see Fig. 1).

\section{Participants}

Participants within each study site include ED staff and physicians, CMHA clinicians, and administrators, as well as patients (and their caregivers) presenting with $\mathrm{MH}$ concerns. Site champions will be identified for optimal local implementation.

\section{Intervention strategy}

As illustrated in Fig. 2, our intervention strategy will build on core components with findings from site visits and qualitative interview findings to identify relevant behavior change techniques for our tailored intervention strategy.

\section{Core components}

Based on experience in related provincial and regional implementations [12-15], we have provisionally selected core intervention components, as listed in Table 1. Engagement of respected and influential champions at each site is a critical element in promoting and maintaining local interest in the pathway. Additional components include education sessions, website support, posters, and reminders. Currently, an implementation toolkit for the EDMHCP is readily available online (http://www.pcmch. on.ca/health-care-providers/paediatric-care/pcmch-strate 


\section{Core Components $+$ Tailoring: Qualitative Interviews $\downarrow$ \\ Final Tailored Strategy}

Fig. 2 Intervention strategy

gies-and-initiatives/ed-clinical-pathways/) [8]. These instructions, measures, and training materials will be tailored for each site and hosted online during the implementation phase on the HEADS-ED website [16]. While the study team will provide guidance and ongoing support, site members will deliver relevant activities to ensure a realistic assessment of requirements for this and future implementations.

\section{Theory-based design with knowledge user input}

Further aspects of our intervention strategy will be based on the Theoretical Domains Framework (TDF), which consists of a set of conceptual determinants and associated theoretical constructs that are believed to influence behavior and stimulate behavior change [17, 18]. The TDF provides a useful approach to identifying relevant behavioral determinants and designing interventions that will address these [19]. We have successfully conducted TDF-based focus groups with various urban and rural ED

Table 1 Implementation strategy core components

\begin{tabular}{ll}
\hline Core component & Description \\
\hline $\begin{array}{l}\text { Local site champion } \\
\text { teams }\end{array}$ & $\begin{array}{l}\text { ED physician or staff (nurse educator, ED nurse) } \\
\text { CMHA clinician or administrator }\end{array}$ \\
Hospital and CMHA & Facilitation through hospital approval processes \\
Commitment & Prioritization within other hospital initiatives \\
Site visits & $\begin{array}{l}\text { Assessment of local ED culture, organization, } \\
\text { and feedback on clinical pathway usability } \\
\text { (human design factor analysis) }\end{array}$ \\
Ongoing site support & $\begin{array}{l}\text { Bimonthly teleconferences with site teams } \\
\text { Education }\end{array}$ \\
Train-the-trainer workshops \\
HeADS-ED training videos \\
Posters/reminders & Resource materials \\
\hline
\end{tabular}

teams on implementing the HEADS-ED screening tool [7], an integral component of the EDMHCP. For this study, additional TDF focus groups, with up to 10 participants, will be conducted at each CMHA to elicit perceptions related to adopting the EDMHCP. Further guiding our strategy, we will assess organizational readiness and relevant factors for change through TDF-guided key informant (KI) interviews with one administrator at each site. All interviews will be audiotaped and transcribed for analysis.

\section{Site visits}

Implementation will begin with site visits to further assess organizational issues within CMHAs and EDs, such as intake and flow of pediatric patients, specific and shared roles of health providers, and pathway fit and acceptability. Infrastructure requirements and implementation readiness will also be assessed. A structured site visit form will be used to take notes and guide visits at each ED or CMHA.

\section{Intervention mapping}

We will conduct a mapping exercise to link relevant behavioral determinants among health providers identified through the qualitative interviews. We will then tailor our core strategy by applying known taxonomies of behavior change techniques $[20,21]$ to identify and select appropriate modes of delivery for a multifaceted intervention strategy that would go beyond simply educating for change. For example, methods may include facilitation, nudging, or systems change. Feasibility and practicality will be important considerations to ensure implementation success.

\section{Ongoing support and communication}

Ongoing support will be provided to expedite pathway implementation and mitigate delays due to other organizational priorities. We will communicate monthly with site champions to support progress during implementation and every 2 months in the post-implementation phase to ensure sustainability. The project coordinator will communicate regularly to ensure target dates are met, using a process log to track reasons for delays that will be explored further in the post-implementation interviews.

\section{Project phases}

The project phases and designated timelines are outlined in Table 2. Once REB approval is secured at each site, the pre-implementation phase will proceed with the site readiness visits and qualitative interviews that will determine the final tailored intervention strategies. The implementation phase for each dyad begins with a process to secure a written memorandum agreement between the ED and CMHA to ensure clarity of responsibilities and expectations integral to the pathway. Implementation will proceed as per the tailored intervention 
Table 2 Description of project phases

\begin{tabular}{|c|c|c|}
\hline Project phase & Duration & Activities \\
\hline \multirow[t]{6}{*}{ Pre-implementation } & \multirow[t]{6}{*}{6 months } & $\begin{array}{l}\text { Site recruitment and REB } \\
\text { approvals }\end{array}$ \\
\hline & & $\begin{array}{l}\text { Project launch team } \\
\text { meeting }\end{array}$ \\
\hline & & Site readiness visits \\
\hline & & $\begin{array}{l}\text { Qualitative interviews } \\
\text { and analysis }\end{array}$ \\
\hline & & Intervention development \\
\hline & & Site champion training \\
\hline \multirow[t]{4}{*}{ Implementation } & \multirow{4}{*}{$\begin{array}{l}17 \text { months } \\
\text { (8 months per dyad) }\end{array}$} & Site customization \\
\hline & & Forms approvals \\
\hline & & Clinical pathway training \\
\hline & & Adoption and feedback \\
\hline \multirow[t]{2}{*}{ Post-implementation } & \multirow[t]{2}{*}{12 months } & $\begin{array}{l}\text { Post-implementation } \\
\text { site visits }\end{array}$ \\
\hline & & $\begin{array}{l}\text { Qualitative interviews } \\
\text { and analysis }\end{array}$ \\
\hline \multirow{3}{*}{$\begin{array}{l}\text { Data collection and } \\
\text { analysis }\end{array}$} & \multirow[t]{3}{*}{4 months } & Chart audits \\
\hline & & $\begin{array}{l}\text { Chart to administrative } \\
\text { database linkage }\end{array}$ \\
\hline & & Quantitative data analysis \\
\hline \multirow[t]{2}{*}{ Follow-up } & \multirow[t]{2}{*}{2 months } & $\begin{array}{l}\text { Project partner meeting: } \\
\text { review of findings }\end{array}$ \\
\hline & & Wrap-up and dissemination \\
\hline
\end{tabular}

strategy. Completed pathway implementation requires, at a minimum, the following: EDMHCP site customization and committee approvals, delivery of at least two educational workshops for each organization, and EDMHCP availability in the ED. We have designated an 8-month period for EDMHCP implementation within each dyad. Following initiation within the first dyad, subsequent dyads will begin implementation in a staggered pattern every 3 months to allow for optimal study team support and incorporating learning from experience with previous dyads. In the post-implementation phase, qualitative interviews will explore experience with the implementation and site audits will be done to assess implementation status. We have defined the data collection periods as follows: (i) post-implementation, as the consecutive 9-month span that follows implementation completion plus an initial 3-month "settling in" time and (ii) preimplementation, as a similar 9-month span prior to the intervention.

\section{Evaluation}

To assess the impact of the EDMHCP implementation and explore the factors leading to our eventual findings, we have planned a mixed methods evaluation approach employing both quantitative and qualitative evaluations, as summarized in Table 3 and described further below. Additionally, a concurrent process evaluation will be conducted to assess the degree of variability and fidelity in implementation of the intervention within each dyad. This evaluation will include a process log, postimplementation site visits, and qualitative interviews.

\section{Outcome measures}

Table 4 provides a summary of study outcomes with the corresponding evaluation measures. The primary process outcome is the proportion of patients with documented $\mathrm{MH}$-specific recommendations, as defined by the project team and based on the EDMHCP, in the medical chart. The primary $\mathrm{MH}$ service outcome is the proportion of patients that receive the EDMHCP recommended follow-up: either within $24 \mathrm{~h}$ or 7 days post-ED visit, based on pathway defined risk level. Recognizing logistic and scheduling factors, a successful outcome will permit a 12-h or 3-day window for follow-up. Adherence, within-window adherence, and non-adherence will be analyzed separately. Secondary outcomes include (1) EDMHCP uptake in the ED, measured as the proportion of completed clinical pathway forms filed in the health record; (2) post-ED uptake of recommended community $\mathrm{MH}$ services, as measured by the Services for Children and Adolescent-Parent Interview (SCA-PI) tool [21]; (3) alignment of recommended services to the HEADS-ED assessment; (4) ED length of stay, hospital admissions, ED revisits (10 days and 3 months) obtained from health records and National Ambulatory Care Reporting System (NACRS) data; and (5) patient/caregiver satisfaction with the ED visit as measured by the Services for Children and Adolescent-Parent Interview (SCA-PI) [22] and the Client Satisfaction Questionnaire (CSQ-8) [23].

\section{Health record audits}

To evaluate whether the intervention results in EDMHCP use among ED clinicians and documentation of $\mathrm{MH}$ specific discharge recommendations, we will audit health records of relevant patients seen during alternate weeks in each 9-month pre- and post-implementation period. ICD10 codes (F codes, mental, and behavior disorders; $\mathrm{X}$ codes, intentional self-poisoning and self-harm; Y codes, poisoning and self-harm of undetermined intent) listed as

Table 3 Evaluation components

\begin{tabular}{ll}
\hline Quantitative evaluation & Qualitative evaluation \\
\hline Patient chart audits & Patient surveys \\
Administrative data & Process evaluation \\
Patient surveys & Process log \\
& Post-implementation \\
& Site visits \\
& Key informant interview \\
& Focus groups \\
\hline
\end{tabular}


Table 4 Study outcomes: process and service

\begin{tabular}{|c|c|c|c|c|}
\hline \multicolumn{2}{|l|}{ Outcome measure } & Description & Details & Data source \\
\hline \multirow[t]{2}{*}{ Process outcomes } & $1^{\circ}$ & Documented $\mathrm{MH}$ recommendations & $\begin{array}{l}\text { Proportion of patients with documented } \mathrm{MH} \text {-specific } \\
\text { recommendations in the medical chart }\end{array}$ & Health record audits \\
\hline & $2^{\circ}$ & EDMHCP uptake in the ED & $\begin{array}{l}\text { Measured as the proportion of completed CPs filed } \\
\text { in health records }\end{array}$ & Health record audits \\
\hline \multirow[t]{9}{*}{ Service outcomes } & \multirow[t]{3}{*}{$1^{\circ}$} & \multirow[t]{3}{*}{ Patients receiving post-ED follow-up } & \multirow{3}{*}{$\begin{array}{l}\text { Proportion of patients that receive post-ED follow-up } \\
\text { as per EDMHCP recommendations }\end{array}$} & Health record audits \\
\hline & & & & NACRS \\
\hline & & & & Telephone follow-up \\
\hline & \multirow[t]{6}{*}{$2^{\circ}$} & $\begin{array}{l}\text { Patient perspectives of post-ED MH } \\
\text { service uptake }\end{array}$ & $\begin{array}{l}\text { Post-ED uptake of recommended community MH } \\
\text { services }\end{array}$ & $\begin{array}{l}\text { Services for Children and } \\
\text { Adolescent-Parent Interview } \\
\text { (SCA-PI) }\end{array}$ \\
\hline & & \multirow{2}{*}{$\begin{array}{l}\text { Alignment of risk assessment with } \mathrm{MH} \\
\text { services recommendations }\end{array}$} & \multirow{2}{*}{$\begin{array}{l}\text { Alignment of recommended services to the } \\
\text { HEADS-ED assessment }\end{array}$} & Health record audits \\
\hline & & & & NACRS \\
\hline & & \multirow[t]{2}{*}{ Hospital metrics } & \multirow{2}{*}{$\begin{array}{l}\text { ED LOS, hospital admissions, ED revisits } \\
\text { (10 days, } 3 \text { months) }\end{array}$} & Health record audits \\
\hline & & & & NACRS \\
\hline & & Patient satisfaction & Patient/caregiver satisfaction with the ED visit & $\begin{array}{l}\text { Client Satisfaction } \\
\text { Questionnaire (CSQ-8) }\end{array}$ \\
\hline
\end{tabular}

MH mental health, EDMHCP Emergency Department Mental Health Clinical Pathway, NACRS National Ambulatory Care Reporting System, ED LOS emergency department length of stay

primary or secondary diagnoses will identify relevant patient charts during the study period. Abstracted data will include demographic and sufficient clinical data to determine risk assessment, disposition plans, and adherence with EDMHCP recommendations. Two auditors, blinded to the study aims and protocol, will be trained to abstract and directly enter health records data into REDCap, an online database [24]. A data dictionary will be created to guide auditors and ensure standardized data collection procedures. Auditors will each abstract the same 50 charts to assess inter-rater agreement. This will be measured for key variables with a kappa coefficient and further training will be done until a prevalence and bias-adjusted kappa $>0.6$ is achieved.

Based on historical administrative data, the number of pediatric $\mathrm{MH}$ visits at the pediatric specialized center is expected to be approximately 2250 during each 9-month period. This sample size will permit estimation of a proportion at this site to within $\pm 2.1 \%$, conservatively assuming a true proportion of $50 \%$. At the other extreme, the lowest number of pediatric $\mathrm{MH}$ visits during each 9month period among the other three sites is expected to be approximately 61 at the rural hospital, which will permit estimation of a proportion to within $\pm 12.5 \%$. Pooling across sites, the total sample size in each 9-month period is anticipated to be approximately 2503, which will permit estimation of a proportion to within $\pm 2.0 \%$.

\section{Administrative databases}

Administrative databases will be used to evaluate whether the pathway improves health care coordination and decreases subsequent ED utilization and wait times. We will use NACRS data from each hospital to assess
ED length of stay, hospital admissions, and number and frequency of repeat ED visits at 10 days and 3 months post the index ED visit. Data will be prospectively collected into a shared ED-CMHA database tool to assess CMHA service times (intake, full assessment and treatment) post-ED visit.

\section{Health consumer surveys}

Patient/caregiver surveys will be used to assess satisfaction with the ED visit and service within CMHAs. Because the EDMHCP recommends CMHA referral intake within 7 days, we will use structured interviews and validated measures to conduct a 10-day post-ED visit survey to assess ED visit satisfaction and whether CMHA intake has occurred. Patients or their caregivers will be contacted by e-mail, post, or phone within 7-10 days of the ED visit and will be given three follow-up measures by phone: (1) structured interview to determine if patients had initiated and received recommended $\mathrm{MH}$ services 10 days after being discharged from the ED; (2) the eight-item Client Satisfaction Questionnaire (CSQ-8) to determine patients' satisfaction with heath care services received in the ED as reported by caregivers or youth; and (3) The Services for Children and AdolescentParent Interview (SCA-PI) which describes pediatric $\mathrm{MH}$ services received across multiple settings, as reported by caregivers. To reduce respondent burden, for the purpose of this study, only eight questions concerning services received for $\mathrm{MH}$ issues have been selected. The SCA-PI will be used to measure patients' use of community resources and document perceptions of the ED's role in connecting them to $\mathrm{MH}$ services. 


\section{Process evaluation}

To explore reasons for potential variation across in implementation effectiveness across the study sites, we will conduct a concurrent process evaluation by documenting characteristics of our intervention, as well as the participants' response to the delivery. This evaluation will assess the degree of variability and fidelity in implementation across the sites, revealing the degree to which the intervention addressed the relevant factors for change. Specific components of this process evaluation will include process logs, data from the post-implementation site visits, and qualitative interviews. Reasons for lack of completed implementation, should this occur at any site, will be detailed and further explored in the postimplementation phase interviews. Lessons learned will be documented and shared with site partners to promote sustained pathway use.

\section{Process log}

A process log will be used to longitudinally track key outcomes and capture issues related to site customization of documents, barriers and delays, workshop attendance and interest, ease of use, and degree of pathway uptake. Log components will include interim survey feedback, monthly updates from site champions, and implementation progress with negotiated target dates. Additional site support will be provided as needed, based on interim findings.

\section{Site visits}

Two months following completed implementation, structured site visits will be conducted to assess accessibility and operational knowledge regarding the pathway. Findings will be compared to pre-implementation visits for each site.

\section{Qualitative interviews}

To explore organizational issues, team dynamics and other relevant issues impacting EDMHCP implementation, post-implementation interviews will be held using TDF-based interview guides. On-site focus groups will be held with up to ten participants per organization. The focus group moderator will record field note observations. As well, KI interviews will be conducted with an administrative lead for each organization. Additional focus group sessions or KI interviews will be held if data saturation is not achieved. All interview sessions will be audiotaped and transcribed for analysis.

\section{Analysis}

\section{Quantitative analysis}

Descriptive statistics (means and standard deviations for continuous variables or medians with interquartile ranges) will summarize EDs, CMHAs, and patient characteristics.
Frequencies and proportions for categorical variables will be reported. We will use a multiple baseline study design and conduct interrupted time-series analysis using segmented regression models, adjusting for site, with ARIMA autocorrelation structure [25-27] to evaluate whether the EDMHCP has resulted in improved health care utilization, medical management, and health sector coordination. Two-sided $p$ values less than 0.05 will be considered statistically significant. Model estimates will be tabulated, together with $95 \%$ confidence intervals. We will also calculate hospital level summary measures including proportions in the pre- and post-intervention periods at each site and referred to each CMHA. An unweighted mean of the change from pre- to post-intervention will be calculated for each hospital ED. The main effect measure will be calculated as the difference between the mean changes in intervention.

Qualitative analysis is integral to determine relevant factors and experience with EDMHCP use. Pre/post-site visits, focus groups, and KI interviews will yield complex data that will inform the intervention and prepare for the EDMHCP implementation (pre) and verify EDMHCP uptake status and assess stakeholders' experiences regarding the intervention success. Interviews will be audiotaped and transcribed. Using the TDF, two coders will independently analyze all transcripts and field notes. The analyses will be provided in an overall thematic format prior to the intervention and 6-month post-intervention. To monitor progress and pursue emerging themes, data collection and analysis will proceed iteratively and concurrently [28]. The qualitative analyst will feed relevant emerging themes back to the interviewer who will revise the interview guide as needed to capture new ideas. Inductive analysis will be managed using N-Vivo10 software and will occur in three phases: coding, using the TDF [18], and categorizing/developing themes. For consistent application, codes will be operationally defined and placed into broad categories that correspond to the major units of analysis. As categories emerge, their theoretical properties will be defined. Comparisons between multiple categories will be conducted to locate similarities and differences between them. Categories will then be synthesized into themes.

\section{Ethics and registration}

Ethics approval for this study has been granted at the coordinating site (Children's Hospital of Eastern Ontario Research Ethics Board - CHEOREB\# 15/146X). Prior to implementation within each dyad, REB approval will be obtained for each site hospital and CMHA. Informed consent will be sought for interviews and focus group meetings. All research data will be stored on a secure server. This trial is registered with ClinicalTrials.gov (NCT02590302). 


\section{Trial status}

This project is currently in the pre-implementation phase, seeking local ethics approvals and data sharing agreement, and preparing the intervention strategy.

\section{Discussion}

By operationalizing best evidence into ED practices and formulating ED-CMHA partnerships through explicit agreements, the EDMHCP is poised to address current system gaps in addressing mental health for youth. This clinical pathway will standardize language and care provided through improved communication and explicit expectations for transition between ED and CMHA settings. Our evaluation will also rigorously address requirements for success and inform scalability to other settings. Our research will identify effective strategies to inform EDMHCP adoption in any community and provide knowledge on its impact by evaluating service integration improvements for this vulnerable population.

While trials are more typically conducted with a complete intervention strategy specified in advance, the unique nature of our proposed implementation requires rich understanding of professional and system issues within each organizational context. Hence, the importance of incorporating findings from our qualitative interviews and site visits to optimize likelihood of implementation success.

Important aspects of our tailored intervention strategy include opportunities for providers at each site to identify strengths and barriers to EDMHCP implementation and the deliberate plan to include site leads in the modification process from the beginning. The planned site visits, qualitative interviews, and continued communication will be helpful in generalizing the pathway implementation to other ED and CMHA settings of various sizes, staff, and urban/rural locations. Further, the tailored resources hosted on the HEADS-ED website [16] will be helpful reinforcement for sites that experience a high staff turnover, particularly for new ED residents and staff.

Given the complexity and novel aspects of this intervention, we anticipate some challenges in promoting modifications to current workflows. Each dyad contains the often hectic and somewhat unpredictable ED environment and the variability among CMHAs in their capacity and structure. Additionally, EDs and CMHAs do not typically interact closely, despite the shared patient population that moves between their settings. Other real-life issues such as budget restrictions, staff turnover, and competing project priorities have also been considered in setting our project timelines. At this stage, these issues are not prohibitive for any of our proposed study sites; however, this is always subject to change. Such is the challenge of implementation research.

Our study findings will be relevant for health systems and professionals responsible for ensuring standardized, quality care for children, and youth with acute $\mathrm{MH}$ concerns. To ensure our findings directly impact relevant service delivery areas, we have specifically recruited study team members with decision-making authority and/or influence on delivery of care for children and youth with $\mathrm{MH}$ concerns. We expect the protocol and findings can be customized and implemented in other EDs and CMHA settings. Equally important, this project will contribute further to implementation evidence relevant to use of clinical pathways and use of theory-based change strategies.

\section{Abbreviations}

CHEO, Children's Hospital of Eastern Ontario; CMHA, community mental health agency; CSQ-8, client satisfaction questionnaire; ED, emergency department; EDMHCP, Emergency Department Mental Health Clinical Pathway; HEADS-ED, screening tool that includes home, education, activities, drugs, suicidality, emotions, and discharge resources; Kl, key informant (interviews); $\mathrm{MH}$, mental health; NACRS, National Ambulatory Care Reporting System; REB, Research Ethics Board; SCA-PI, services for children and adolescents-parent interview; TDF, Theoretical Domains Framework

\section{Acknowledgements}

We are grateful to the Canadian Institutes of Health Research (CIHR) and the Ministry of Health and Long-Term Care (MOHLTC) for their financial support of this project. We would like to acknowledge Nathalie Thibedeau for her contributions to the original grant submission. We would also like to thank our hospital site partners, community agency partners, and knowledge users for their letters of support.

\section{Funding}

The funding source in support of this protocol includes a CIHR (\#337794) and MOHLTC (\#6719) Partnerships for Health Systems Improvement Grant, June 2015 to June 2018. These funding bodies had no role in the study design, data collection, analysis, interpretation, nor writing of the manuscript.

\section{Authors' contributions}

MJ co-chaired the provincial working group to develop this ED clinical pathway. MJ's clinical pathway protocol [10] was adapted for the current study. MJ, MC, PC, RZ, SR, AK, CG, RZ, and WG contributed to the adaptation of the clinical protocol and the design of the study. CP drafted of the manuscript based on the original protocol. NB conducted the sample size calculations. All authors read, contributed to, and approved the final manuscript.

\section{Authors' information}

The team includes clinical and research expertise in emergency department clinical pathways (MJ), emergency medicine (MJ, SR, RZ), community- and hospital-based pediatric MH (MC, PC, KP, AK, CG, RZ, CP), biostatistics and statistical methodology (NB, WG), scoring tool development (MC, PC, RZ, $A K, C G, W G)$, emergency outreach (SR), community outreach (AK, KP), and knowledge translation (MJ, MC, PC, AK, KP, RZ, CP).

\section{Competing interests}

The authors declare that they have no competing interests.

\section{Ethics approval and consent to participate}

Ethics approval for this study has been granted at the coordinating site (Children's Hospital of Eastern Ontario Research Ethics Board - CHEOREB\# 15/146X). Prior to implementation within each dyad, REB approval will be obtained for each site hospital and CMHA. Informed consent will be sought for interviews and focus group meetings.

\section{Author details}

${ }^{1}$ Division of Emergency Medicine, Department of Pediatrics, Children's Hospital of Eastern Ontario, Ottawa, ON K1H 8L1, Canada. ${ }^{2}$ Faculty of Medicine, Department of Pediatrics, University of Ottawa, Ottawa, Canada. 
${ }^{3}$ Psychiatric \& Mental Health Research, CHEO Research Institute, Ottawa, Canada. ${ }^{4}$ Department of Psychology, Carleton University, Ottawa, Canada. ${ }^{5}$ Centre for Pediatric Mental Health Services and Policy Research, CHEO Research Institute, Ottawa, Canada. ${ }^{6}$ Department of Epidemiology, University of Ottawa, Ottawa, Canada. ${ }^{7}$ Psychology, Children's Hospital of Eastern Ontario, Ottawa, Canada. ${ }^{8}$ Department of Psychiatry, University of Ottawa, Ottawa, Canada. ${ }^{9}$ Psychiatry, Children's Hospital of Eastern Ontario, Ottawa, Canada. ${ }^{10} \mathrm{Clinical}$ Research Unit, CHEO Research Institute, Ottawa, Canada.

${ }^{11}$ Department of Statistics, University of Ottawa, Ottawa, Canada.

${ }^{12}$ Department of Psychology, University of Ottawa, Ottawa, Canada.

Received: 9 June 2016 Accepted: 18 June 2016

Published online: 07 July 2016

\section{References}

1. Kataoka SH, Zhang L, Wells KB. Unmet need for mental health care among U.S. children: variation by ethnicity and insurance status. Am J Psychiat. 2002;159(9):1548-55. doi:10.1176/appi.ajp.159.9.1548.

2. Hacker K, Arsenault L, Franco I, et al. Referral and follow-up after mental health screening in commercially insured adolescents. J Adolesc Health. 2014;55:17-23. doi:10.1016/j.jadohealth.2013.12.012.

3. Kirby MJL, Keon WJ. Out of the shadows at last: transforming mental health mental illness and addiction services in Canada. The Standing Senate Committee on Social Affairs, Science and Technology; 2006.

4. MHASEF Research Team. The mental health of children and youth in Ontario: a baseline scorecard. Toronto: Institute for Clinical Evaluative Sciences; 2015. (Online) Accessed 15 Mar 2016. ISBN 978-1-926850-55-9.

5. Children's Hospital of Eastern Ontario. Advocacy Mental Health. Ottawa, Canada: http://www.cheo.on.ca/en/mentalhealth. Accessed 4 July 2016.

6. Richman B, Grossman D, Sloan F. Fragmentation in mental health benefits and services: a preliminary examination into consumption and outcomes. In: Elhauge ER, editor. The fragmentation of US healthcare: causes and solutions. Toronto: Oxford University Press; 2010. p. 279-300. http://dx.doi.org/10.2139/ssrn.1460935.

7. MacWilliams K, Curran J, Racek J, et al. Barriers and facilitators to implementing the HEADS ED: a rapid screening tool for pediatric patients in emergency departments. Pediatr Emerg Care. 2016. doi:10.1097/PEC.0000000000000651.

8. Provincial Council for Maternal and Child Health. Implementation toolkit: emergency department clinical pathway for children and youth with mental health conditions. Prov Counc Matern Child Heal. Available at: http://www.pcmch.on.ca/wp-content/uploads/2015/09/1.0-Toolkit-ED_ Clinical_Pathway-Tookit_Oct_29_2014_Updated-PPO.pdf. Accessed 4 July 2016.

9. Cappelli M, Gray C, Zemek R, et al. The HEADS-ED: a rapid mental health screening tool for pediatric patients in the emergency department. Pediatric. 2012;130(2):e321-7. doi:10.1542/peds.2011-3798.

10. Jabbour M, Curran J, Scott SD, et al. Best strategies to implement clinical pathways in an emergency department setting: study protocol for a cluster randomized controlled trial. Implement Sci. 2013;8(1):55. doi:10.1186/1748-5908-8-55.

11. Schulz KF, Altman DG, Moher D, et al. CONSORT 2010 statement: updated guidelines for reporting parallel group randomized trials. Ann Int Med. 2010;152(11):726-32. doi:10.7326/0003-4819-152-11-201006010-00232.

12. Scott SD, Grimshaw J, Klassen TP, et al. Understanding implementation processes of clinical pathways and clinical practice guidelines in pediatric contexts: a study protocol. Implement Sci. 2011;6:133. doi:10.1186/1748-5908-6-133.

13. Jabbour $\mathrm{M}$, Reid $\mathrm{S}$, Irwin $\mathrm{D}$, et al. Clinical pathway-based pediatric emergency outreach program: implementation and preliminary evaluation. Can J Hosp Pharm. 2014;67(3):240-5. http://dx.doi.org/10.4212/cihp.v67i3.1362. Accessed 4 July 2016.

14. Lougheed MD, Olajos-Clow J, Szpiro K, et al. Multicentre evaluation of an emergency department asthma care pathway for adults. CIEM. 2009;11(3):215-29.

15. Bhogal S, Bourbeau J, McGillivray D, et al. Adherence to pediatric asthma guidelines in the emergency department: a survey of knowledge, attitudes and behaviour among health care professionals. Can Respir J. 2010;17:175-82. http://dx.doi.org/10.1155/2010/274865. Accessed 4 July 2016.

16. HEADS-ED, From screening to services. www.ottawa.heads-ed.com. Accessed 4 July 2016.

17. Michie S, Johnston M, Abraham C, et al. Making psychological theory useful for implementing evidence based practice: a consensus approach. Qual Saf Healthc. 2005;14(1):26-33. http://dx.doi.org/10.1136/qshc.2004.011155. Accessed 4 July 2016.

18. Cane J, O'Connor D, Michie S. Validation of the theoretical domains framework for use in behaviour change and implementation research.
Implement Sci. 2012;7(37):1-17. http://dx.doi.org/10.1186/1748-5908-7-37. Accessed 4 July 2016.

19. Michie S, Johnston M, Francis J, et al. From theory to intervention: mapping theoretically derived behavioral determinants to behaviour change techniques. Appl Psychol. 2008;57(4):660-80. doi:10.1111/j.1464-0597. 2008.00341.x.

20. Michie $S$, van Stralen MM, West R. The behaviour change wheel: a new method for characterizing and designing behaviour change interventions. Implement Sci. 2011;6(42):1-11. doi:10.1186/1748-5908-6-42.

21. Waltz TJ, Powell BJ, Matthieu MM, et al. Use of concept mapping to characterize relationships among implementation strategies and assess their feasibility and importance: results from the Expert Recommendations for Implementing Change (ERIC) study. Implement Sci. 2015;10(109):1-8. doi:10.1186/s13012-015-0295-0.

22. Jensen PS, Eaton Hoagwood K, Roper M, et al. The services for children and adolescents-parent interview: development and performance characteristics. J Am Acad Child Adolesc Psychiat. 2004;43(11):1334-44. doi:10.1097/01.chi. 0000139557.16830.4e

23. Attkisson CC, Zwick RJ. The client satisfaction questionnaire: psychometric properties and correlations with service utilization and psychotherapy outcome. Eval Prog Plann. 1982;5(3):233-7.

24. Harris PA, Taylor $R$, Thielke $R$, et al. Research electronic data capture (REDCap) - a metadata-driven methodology and workflow process for providing translational research informatics support. J Biomed Inform. 2009;42(2):377-81. doi:10.1016/j.jbi.2008.08.010.

25. Hartmann DP, Gottman JM, Jones RR, et al. Interrupted time-series analysis and its application to behavioural data. J Appl Behav Anal. 1980;13(4):543-59.

26. Wagner AK, Soumerai SB, Zhang F, Ross-Degnan D. Segmented regression analysis of interrupted time series studies in medication use research. J Clin Pharm Ther. 2002;27(4):299-309. doi:10.1046/j.1365-2710.2002.00430.x.

27. Zhang F, Wagner AK, Soumerai SB, Ross-Degnan D. Methods for estimating confidence intervals in interrupted time series analysis of health interventions. J Clin Epidemiol. 2009;62(2):143-8. doi:10.1016/j.jclinepi.2008.08.007.

28. Morse J, Field P. Qualitative research methods for health professionals. 2nd ed. Thousand Oaks: Sage; 1995.

\section{Submit your next manuscript to BioMed Central and we will help you at every step:}

- We accept pre-submission inquiries

- Our selector tool helps you to find the most relevant journal

- We provide round the clock customer support

- Convenient online submission

- Thorough peer review

- Inclusion in PubMed and all major indexing services

- Maximum visibility for your research

Submit your manuscript at www.biomedcentral.com/submit
) Biomed Central 